Field : Sport Sciences

Type : Research Article

Recieved:05.10.2015 - Accepted:23.11.2015

\title{
Can a 5 Week Strength Training Program Produce Range of Motion Adaptations in Amateur Female Tennis Players?
}

\author{
Andrew L. SHIM, Yvonne NIEDERBRACHT, Hal STROUGH \\ Department of Kinesiology \& Human Performance, Briar Cliff University, Sioux City, IA., Mathias- \\ Spital Rheine, Germany. The College of St. Scholastica, Duluth, MN \\ Email: andrew.shim@briarcliff.edu
}

\begin{abstract}
Objective: The purpose of this study was to assess range of motion adaptations in amateur tennis players based on the effects of a five week strength training program on the dominant and non-dominant arm. Subjects: An experimental and control group of six collegiate women tennis players (Div II and NAIA) participated. After initial assessment, six subjects participated in a five week, four times a week, pre-season strength training program consisting of five exercises: External Rotation $90^{\circ}$, Seated Row, Scaption, Chest Press, and External Shoulder Rotation (Rubber tubing). Results: Data analysis through a paired t-test showed that there were no significant changes in ROM in the experimental group when compared to the control group. In conclusion, a strength training program is highly recommended for female overhead athletes combined with a proper flexibility regimen to promote best practice.
\end{abstract}

Keywords: Tennis, Female Athletes, Range of Motion, Strength Training 


\section{Introduction}

Tennis requires high biomechanical demands upon the musculoskeletal system (Patel et al., 2002) as well as great deal of neuromuscular coordination, speed, agility, and power. Roetert et al. (2009) noted that primary kinetic chain motions creating forehand racket speed are horizontal shoulder adduction, trunk rotation, and internal rotation. In addition, strength, agility, power, speed, and explosiveness are associated with tournament performance (Fernandez-Fernandez et al., 2009). Racket speeds can generate velocities of up to 70 to 80 miles per hour and ball speeds of 80 to 115 miles per hour in high-level competitions. Theses forces cause adaptive changes in maximum strength of the internal and external shoulder rotator muscles and range of motion, potentially putting the athlete at increased risk for injury (Knapik et al., 1991; Kibler et al., 1996; Ellenbecker \& Roetert, 2003).

Studies on elite junior and collegiate tennis players (Chandler et al., 1992) have found that internal rotational torque and power of the dominant arm, but not external rotational torque and power, were significantly increased as compared to internal rotation torque and power on the non-dominant arm. Male concentric external rotation / concentric internal rotation total work ratios ranged between $64-72 \%$ on the dominant arm and $77-84 \%$ on the non-dominant arm. Female ratios for the dominant arm were similar to those measured in the male subjects, ranging between $61-76 \%$, and were slightly higher on the non-dominant arm, ranging between 77 and 120\% (Ellenbecker \& Roetert, 2003).

Chepaha (2011) observed 37 male and female overhead student-athletes over a period of four and eight weeks and discovered internal and external rotator deficits in range of motion in both dominant and non-dominant limbs. Male and female tennis players showed $10 \%$ deficits in their shoulder range of motion without the implementation of a stretching or strength program during this period. Rotert et al., (2000) studied 44 nationally ranked junior tennis players and showed significant decreases in the range of motion between the non-dominant limb compared to their dominant side with internal rotation of the shoulder. In the younger age groups of 15 or less, the differences were very minimal. Ellenbecker \& Rotert (2002) looked at the effects of strength training and range of motion on female collegiate tennis players but only with shoulder flexion and extension and found no significance with range of motion with both limbs after four months of training. Moreno-Perez et al., (2015) studied professional tennis players with self-reported shoulder pain to ascertain differences in range of motion compared to asymptomatic controls. The dominant shoulder had reduced internal rotation (IR) and total ROM but increased external rotation (ER) compared to the nondominant side. Tennis players with shoulder pain showed less IR ROM versus no pain group. Could the role of strength training play a valuable component of either maintaining or improving range of motion with internal or external rotation of the shoulder in female amateur tennis players?

The purpose of this study was to assess range of motion adaptations in amateur female tennis players and if the effects of a five week strength training program had any positive results on the range of motion with the dominant and non-dominant arm. 


\section{Methods}

\section{Subject Selection}

Six female varsity tennis players (5 RHD, 1 LHD) from a Division II institution were recruited as subjects for an experimental group, $20.0 \pm 1.8$ years, $7.7 \pm 4.4$ years played, 165.8 $\pm 7.1 \mathrm{~cm}, 71.5 \pm 9.9 \mathrm{~kg}$ ). In addition, 6 female varsity tennis players from an NAIA institution were recruited to act as a control group $(6$ RHD, $18.8 \pm 0.8$ years, $5.7 \pm 1.0$ years played, $162.3 \pm 5.8 \mathrm{~cm}, 63.7 \pm 18.0 \mathrm{~kg}$ ). The two groups did not significantly differ in age, years played, weight or height. None of the subjects had conducted any specific shoulder strength training six months prior to the study. All subjects and coaches were briefed on the risks of the research project before they signed informed consent forms. The study was furthermore approved by the Institutional Review Board of the sponsoring university.

\section{Procedures}

\section{Assessing Range of Motion}

Active shoulder internal and external rotation range of motion was assessed using the KinCom. The subjects were in a seated position with the shoulder abducted at $90^{\circ}$ and the elbow was flexed at $90^{\circ}$. The elbow was secured by a customized support and the hand grabbed onto a grip. The subject's forearm was then passively rotated to maximal external and internal rotation positions (Kibler et al., 1996). Maximal internal and external rotation were recorded when a firm end point was reached at the glenohumeral joint. External rotation measurements were recorded just before the back arched, internal rotation measurements were recorded just before the shoulder lifted from the back of the seat (Kibler et al., 1996).

\section{Strength Training program}

A 5-week, 4 times per week, strength training program according to Roetert et al. (1997) was implemented throughout the pre-season tennis training program among the experimental subject group from a state university. Five specific exercise targeting the external rotators consisted of the following: seated row, scaption, chest press, external rotation theraband, and external rotation with a dumbbell. None of the experimental group had performed any specific training of the shoulder before the onset of the study. The strength training program was started within 3 days after the pre-training assessment. The control group, which included subjects from a local private college, conducted their regular preseason tennis training and did not participate in any strength training. At the completion of the 5-week period, all subjects were post-tested. Post-testing included isokinetic shoulder strength and range of motion analysis as well as body weight measurements. Post-testing for the experimental group was conducted within 3 days after the last strength training session.

Design and Analysis

Paired $\mathrm{t}$ tests (using two-sided alpha $=0.05$ ) were used to compare mean differences between strength of the internal and external shoulder rotator muscles as well as internal and external range of motion on the dominant and non-dominant arm. Results are summarized as means \pm standard deviations. 


\section{Results}

\section{Range of Motion}

Range of motion varied widely between subjects. Seven subjects were found to have impaired internal rotation mobility below 30 degrees (Tables 1 and 2). The tables show that the active ranges of internal and external rotation did not differ between the control and the experimental group at the beginning of the study.

Table 1. Control Group Pre-Assessment Data

Control Group Pre-assessment Data

\begin{tabular}{|l|r|r|r|r|r|r|}
\hline Subject & $\mathbf{4}$ & $\mathbf{1 1}$ & $\mathbf{5}$ & $\mathbf{2}$ & $\mathbf{1 2}$ & $\mathbf{1 7}$ \\
\hline Age & 18 & 18 & 19 & 19 & 20 & 19 \\
\hline Years Played & 6 & 5 & 6 & 7 & 6 & 4 \\
\hline Weight [kg] & 63.8 & 56.8 & 63.1 & 51.2 & 49.1 & 98.4 \\
\hline Height [cm] & 167 & 162 & 168 & 161 & 152 & 164 \\
\hline Dominant Arm & Right & Right & Right & Right & Right & Right \\
\hline Current Shoulder Injury & No & No & No & No & No & No \\
\hline Past Shoulder Injury & No & No & No & No & No & No \\
\hline Shoulder Strength Training & No & No & No & No & No & No \\
\hline Strength Training & Yes & No & Yes & Yes & Yes & Yes \\
\hline & & & & & & \\
\hline Range of motion & & & & & & \\
\hline Left Internal & 33 & 40 & 50 & 37 & 28 & 45 \\
\hline Left External & 120 & 116 & 116 & 117 & 118 & 114 \\
\hline Right Internal & 29 & 35 & 27 & 44 & 31 & 29 \\
\hline Right External & 116 & 118 & 118 & 114 & 99 & 112 \\
\hline
\end{tabular}

Table 2. Experimental Group Pre-Assessment Data

Experimental Group Pre-assessment Data

\begin{tabular}{|c|c|c|c|c|c|c|}
\hline Subject & 9 & 13 & 14 & 10 & 15 & 16 \\
\hline Age & 20 & 18 & 19 & 19 & 21 & 23 \\
\hline Years Played & 9 & 5 & 5 & 5 & 6 & 16 \\
\hline Weight [kg] & 67.8 & 91 & 69.6 & 70.9 & 63.1 & 66.8 \\
\hline Height $[\mathrm{cm}]$ & 157.5 & 179 & 165.1 & 165 & 165 & 163 \\
\hline Dominant Arm & Right & Right & Right & Right & Left & Right \\
\hline Current Shoulder Injury & No & No & No & No & No & No \\
\hline Past Shoulder Injury & No & No & No & No & No & $\begin{array}{l}\text { Yes (6 } \\
\text { Years } \\
\text { Ago) }\end{array}$ \\
\hline Shoulder Strength Training & No & No & No & No & No & No \\
\hline Strength Training & No & No & No & No & No & No \\
\hline \multicolumn{7}{|l|}{ Range of motion } \\
\hline Left Internal & 28 & 43 & 44 & 27 & 28 & 52 \\
\hline Left External & 116 & 115 & 92 & 151 & 152 & 116 \\
\hline Right Internal & 30 & 30 & 32 & 57 & 34 & 42 \\
\hline Right External & 140 & 111 & 97 & 110 & 116 & 127 \\
\hline
\end{tabular}


Table 3. Control Group Post-Assessment

Control Group Post-assessment Data

\begin{tabular}{|l|r|r|r|r|r|r|}
\hline Subject & $\mathbf{4}$ & $\mathbf{1 1}$ & $\mathbf{5}$ & $\mathbf{2}$ & $\mathbf{1 2}$ & $\mathbf{1 7}$ \\
\hline Age & 18 & 18 & 19 & 19 & 20 & 19 \\
\hline Years Played & 6 & 5 & 6 & 7 & 6 & 4 \\
\hline Weight [kg] & 65.7 & 55 & 66.2 & 51.2 & 49.7 & 98.2 \\
\hline Height [cm] & 167 & 162 & 168 & 161 & 152 & 164 \\
\hline Dominant Arm & Right & Right & Right & Right & Right & Right \\
\hline Current Shoulder Injury & No & No & No & No & No & No \\
\hline Past Shoulder Injury & No & No & No & No & No & No \\
\hline Shoulder Strength Training & No & No & No & No & No & No \\
\hline Strength Training & Yes & No & Yes & Yes & Yes & Yes \\
\hline & & & & & & \\
\hline Range of motion & & & & & & \\
\hline Left Internal & 36 & 28 & 42 & 33 & 34 & 35 \\
\hline Left External & 116 & 114 & 131 & 119 & 116 & 124 \\
\hline Right Internal & 38 & 32 & 44 & 33 & 30 & 33 \\
\hline Right External & 107 & 117 & 131 & 121 & 114 & 119 \\
\hline
\end{tabular}

Table 4. Experimental Group Post-Assessment Data

\begin{tabular}{|c|c|c|c|c|c|c|}
\hline Subject & 9 & 13 & 14 & 10 & 15 & 16 \\
\hline Age & 20 & 18 & 19 & 19 & 21 & 23 \\
\hline Years Played & 9 & 5 & 5 & 5 & 6 & 16 \\
\hline Weight [kg] & 67.4 & 92.7 & 70.3 & 72 & 63.9 & 66.5 \\
\hline Height [cm] & 157.5 & 179 & 165.1 & 165 & 165 & 163 \\
\hline Dominant Arm & Right & Right & Right & Right & Left & Right \\
\hline Current Shoulder Injury & No & No & No & No & No & No \\
\hline Past Shoulder Injury & No & No & No & No & No & $\begin{array}{l}\text { Yes ( } 6 \\
\text { Years } \\
\text { Ago) }\end{array}$ \\
\hline Shoulder Strength Training & No & No & No & No & No & No \\
\hline Strength Training & No & No & No & No & No & No \\
\hline \multicolumn{7}{|l|}{ Range of motion } \\
\hline Left Internal & 51 & 42 & 48 & 32 & 28 & 49 \\
\hline Left External & 144 & 118 & 118 & 146 & 133 & 129 \\
\hline Right Internal & 66 & 29 & 29 & 41 & 28 & 51 \\
\hline Right External & 138 & 121 & 121 & 147 & 117 & 126 \\
\hline
\end{tabular}

\section{Discussion}

This study was truly unique based upon the focus on a group of competitive, non-junior, intermediate level, female tennis players, compared to previously researched groups of elite male or mixed gender junior players (Codine, Bernard, Pocholle, Benaim \& Brun, 1997; Ellenbecker, George, Davies, \& Rowinski, 1988; Mont, Cohen, Campbell, Gravare, \& Mathur, 1994; Treiber et al. 1998; Stanley et al., 2004; Moreno-Perez et al., 2015). Observations on the number of years played (Tables 3 and 4), indicate that collegiate teams at the Division II level encompass players from a wide range of tennis playing experiences from five to sixteen years of experience.

\section{Range of Motion}

Alterations in joint range of motion or muscle flexibility are thought to be important concerns with injury risk to athletes, especially in repetitive use sports such as tennis, baseball and 
running (Kibler \& Chandler, 2003). Comparable to results obtained by Roetert and Ellenbecker (1998) and Moreno-Perez (2015), this study observed poor internal flexibility on the dominant arm among all subjects (Tables $1 \& 2$ ). Standard values established by Kibler et al. (1996) found dominant internal range of motion to be $43.3^{\circ}$, dominant external range of motion to be $126.5^{\circ}$, non-dominant internal range of motion to be $72.8^{\circ}$, and non-dominant external range of motion equal to $120.7^{\circ}$ from a group of 19 female tennis players with an average age of 18 years. Overall, internal range of motion measurements obtained in this study were significantly lower than those obtained by Roetert and Ellenbecker (1998) and Kibler et al. (1996). Zusa et al., (2012) also found significant differences between the dominant and non-dominant limbs in internal and external rotation. Their protocol used a different device (Technogym REV-9000) and positioned subjects at 85 degrees of abduction rather than 90. It was not specified whether testing was conducted prior to, during, or following the season. This might be due to the testing protocol being conducted in a seated position, with the elbow fixed in customized support, versus supine with the elbow loose. By isolating the elbow, variation from movement was not possible.

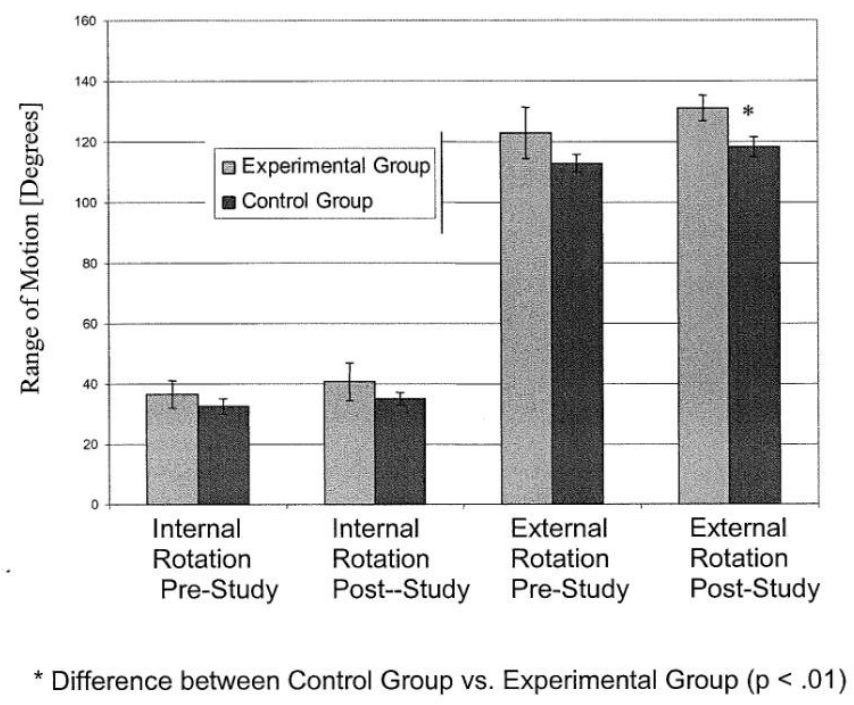

Figure 12. Active ranges of shoulder internal and external rotation in the dominant arm at pre- and post-assessment of the control vs. the experimental group.

Note. Standard error bars depict SD.

Figure 1. Control vs. Experimental Group Range of Motion

Values for external range of motion on the dominant and non-dominant arm were compared to those found in the review of literature. It was interesting to notice that the experimental group tended to have increased external range of motion at post-assessment, which might be due to the stretching effects of some of the strength training exercises, such as seated row and external rotation $90^{\circ}$. To what extent this strength training program influences range of motion around the shoulder joint, however, remains unclear due to the limited sample size. 
This study had similar outcomes as Fernandez-Fernandez et al., (2013) where both experimental and control subjects experienced ROM (IR and ER) increases as a result of completing stretching exercises. The experimental group in this study also performed 3 sessions of approximately one hour each per week consisting of core strength, elastic resistance, and medicine ball. Also, what influence these flexibility adaptations have on the risk of shoulder injury to the overhead athlete is unknown (Wang \& Cochrane, 2001). Marcondes et al. (2013) studied skilled amateur tennis players comparing those asymptomatic with those experiencing pain in their dominant shoulder. There were significant differences between the groups in dominant shoulder ROM, posterior capsule tightness, external rotation strength and strength ratio. This reduction of internal rotation is an absolute loss of motion due to the decrease in total rotation. Kibler et al. (1996) supported that the decrease in shoulder internal rotation can be explained as an adaptation of the posterior shoulder musculature and capsular structures to the tennis stroke. Similarly, the slight increase in shoulder external rotation in tennis players they assume to be a likely adaptation to the tennis serve.

\section{Conclusion}

In conclusion, alterations in joint range of motion or muscle flexibility are thought to be important concerns with injury risk to athletes, especially in repetitive use sports. Past literature has demonstrated that a shoulder specific strength training program could elicit improvements in range of motion in overhead female athletes. This study did not demonstrate this. The sample size, the testing protocols, or possibly the intensity of the training session could have affected the outcome compared to past studies conducted in this specific area. It would be advised that future studies look at improving the intensity of the resistance exercises during throughout the five weeks as well as look at possible alterations with tennis practices to make sure alterations with range of motion could be affected by shoulder musculature and tight joint capsules. Regardless, a flexibility program enhanced by a proper shoulder strength training program is still highly recommended for all overhead activity athletes.

\section{REFERENCES}

Chandler, J. (1995). Exercise training for tennis. Clin Sports Med, 14(1), 33- 46.

Chandler, T. J., Kibler, W. B., Stracener, E. C., Ziegler, A. K., \& Pace, B. (1992). Shoulder strength, power, and endurance in college tennis players. Am J Sports Med, 20(4), 455-8.

Chandler, T. J., Kibler, W. B., Uhl, T. L., Wooten, B., Kiser, A., \& Stone, E. (1990). Flexibility comparisons of junior elite tennis players to other athletes. Am J Sports Med, 18(2), 134-6.

Codine, P., Bernard, P. L., Pocholle, M., Benaim, C., \& Brun, V. (1997). Influence of sports discipline on shoulder rotator cuff balance. Med Sci Sports Exerc, 29(11), 1400-5.

Cohen, D. B., Mont, M. A., Campbell, K. R., Vogelstein, B. N., \& Loewy, J. W. (1994). Upper extremity physical factors affecting tennis serve velocity. Am J Sports Med, 22(6), 74650 . 
Durall, C. J., Manske, R. C., \& Davies, G. J. (2001). Avoiding shoulder injury from resistance training. Strength Cond J, 23(5), 10-18.

Dvir, Z. (1995). Isokinetics: Muscle Testing, Interpretation and Clinical Applications. Churchill Livingstone: Edinburgh, England

Ellenbecker, T. S., Davies, G. J., \& Rowinski, M. J. (1988). Concentric versus eccentric isokinetic strengthening of the rotator cuff. Am J Sports Med, 16(1), 64-9.

Ellenbecker, T. S., \& Roetert, E. P. (1999). Testing isokinetic muscular fatigue of shoulder internal and external rotation in elite junior tennis players. J Ortho Sports Phys Ther, 29(5), 275-81.

Ellenbecker, T. S., \& Roetert, E. P. (2002). Effects of a 4-month season on glenohumeral joint rotational strength and range of motion in female collegiate tennis players. J Strength Cond Res, 6(1), 92-6.

Ellenbecker, T. S., \& Roetert, E. P. (2003). Age specific isokinetic glenohumeral internal and external rotation strength in elite junior tennis players. J Sci Med Sport, 6(1), 63-70.

Fernandez-Fernandez, J., Ellenbecker, T., Sanz-Rivas, D., Ulbricht, A., Ferrauti, A. (2013). Effects of a 6-week junior tennis conditioning program on service velocity. Journal of Sports Science and Medicine, 12, 232-239.

Fernandez-Fernandez, J., Sanz-Rivas, D., Mendez-Villanueva, A. (2009). A review of the activity profile and physiological demands of tennis match play. Strength and Conditioning Journal, 31(4), 15-26.

Gay, L. R., \& Arasian, P. (2003). Educational Research-Competence forAnalysis and Applications, $7^{\text {th }}$ edition. Pearson Education Ltd.: New Jersey.

Harris, M., Williams, C., Stanish, W. D., \& Micheli, L. J. (1994). Oxford Textbook of Sports Medicine. Oxford University Press: Oxford, England.

Kibler, W. B. (1995). Biomechanical analysis of the shoulder during tennis activities. Clin Sports Med, 14(1), 79-84.

Kibler, W. B. (1998). The role of scapula in athletic shoulder function. Am J Sports Med, 26(2), 325-37.

Kibler, W. B., \& Chandler, T. J. (1994). Sport-specific conditioning. Am J Sports Med, 22(3), 424-32.

Kibler, W. B., \& Chandler, T. J. (2003). Range of motion in junior tennis players participating in an injury risk modification program. J Sci Med Sport, 6(1), 51-62.

Kibler, W. B., Chandler, T. J., Livingston, B. P., \& Roetert, E. P. (1996). Shoulder range of motion in elite tennis players: Effect of age and years of tournament play. Am J Sports Med, 24(3), 279-85.

Knapik, J. J., Bauman, C. L., Jones, B. H., Harris, J., \& Vaughan, L. (1991). Preseason strength and flexibility imbalances associated with athletic injuries in female collegiate athletes. American Journal of Sports Medicine, 19(1), 76-81.

Kraemer, W. J., Triplett, N. T., Fry, A. C., Koziris, L. P., Bauer, J. E., \& Lynch, J. M. (1995). An in-depth sports medicine profile of women college players. J Sport Reh, 4, 79-98. 
Kühne, C. A., Zetti, R. P., \& Nast-Kolb, D. (2004). Verletzungs- und Beschwerdehäufigkeit im Tennisleistungs- und Breitensport. [Tennis injuries-pattern and incidence in competitive and leisure tennis sports]. Sportverletz Sportschaden, 18, 85-89.

Marcondes, F.B., deJesus, J.F., Bryk, F.F., de Vasconcelos, R.A, Fukuda, T.Y. (2013). Posterior shoulder tightness and rotator cuff strength assessments in painful shoulders of amateur tennis players. 17(2), 185-194.

McCarrick, M. J., \& Kemp, J. G. (2000). The effect of strength training and reduced training on rotator cuff musculature. Clin Biomech (Bristol, Avon), 15 Suppl 1:S42-5.

Moncrief, S. A., Lau, J. D., Gale, J. R., \& Scott, S. A. (2002). Effect of rotator cuff exercise on humeral rotation torque in healthy individuals. J Strength Cond Res, 16(2), 262-70.

Mont, M. A., Cohen, D. B., Campbell, K. R., Gravare, K., \& Mathur, S. K. (1994). Isokinetic concentric versus eccentric training of shoulder rotators with functional evaluation of performance enhancement in elite tennis players. Am J Sports Med, 22(4), 513-7.

Moreno-Perez, V., Moreside J., Barbado, D., Vera-Garcia, F.J. (2015). Comparison of shoulder rotation range of motion in professional tennis players with and without history of shoulder pain. Manual Therapy, 20, 313-318.

Patel, D. R., Stier, B., \& Luckstead, E.F. (2002). Major international sport profiles. Pediatr. Clin.North Am., 49(4), 769-92.

Regan, D. (1996). The role of scapular stabilization in overhead motion. Strength Cond J, 18(1), 33-7.

Richardson, A. B. (1983). Overuse syndrome in baseball, tennis, gymnastics, and swimming. Clin Sports Med, 2(2), 379-90.

Roetert, E. P., \& Ellenbecker, T.S. (1998). Complete conditioning for tennis. Champaign, IL: Human Kinetics.

Roetert, E. P., Ellenbecker, T. S., Chu, D. A., \& Bugg, B. S. (1997). Tennis-specific shoulder and trunk strength training. Strength Cond J, 19(3), 31-43.

Roetert, E. P., Kovacs, M., Knudson, D., Groppel, J. L. (2009). Biomechanics of the tennis groundstrokes: Implications of strength training. Strength Cond J, 31(4), 41-49.

Sallis, R. E., Jones, K., Sunshine, S., Smith, G., \& Simon, L. (2001). Comparing sports injuries in men and women. Int J Sports Med, 22, 420-23.

Silliman, J. F., \& Hawkins, R. J. (1991). Current concepts and recent advances in the athlete's shoulder. Clin Sports Med, 10(4), 693-705.

Sprigings, E., Marshall, R., Elliott, B., \& Jennings, L. (1994). A three-dimensional kinematic method for determining the effectiveness of arm segment rotations in producing racquet-head speed. J Biomech, 27, 245-54.

Stanley, A., McGann, R., Hall, J., McKenna, L., \& Briffa, N. K. (2004). Shoulder strength and range of motion in female amateur-league tennis players. J Orthop Sports Phys Ther, 34(7), 402-09. 
Treiber, F. A., Lott, J., Duncan, J., Slavens, G., \& Harris, D. (1998). Effects of theraband and lightweight dumbbell training on shoulder rotation torque and serve performance in college tennis players. Am J Sports Med, 26(4), 510-15.

Tyson, A. (1995). Prevention and rehabilitation of shoulder impingement. Strength Cond J, 17(1), 31-4.

Wang, H.-K., \& Cochrane, T. (2001). Mobility impairment, muscle imbalance, muscle weakness, scapular asymmetry and shoulder injury in elite volleyball athletes. J Sports Med Phys Fitness, 41(3), 403-10.

Winge, S., Jørgensen, U., \& Lassen Nielsen, A. (1989). Epidemiology of injuries in danish championship tennis. Int J Sports Med, 10, 368-71.

Zusa, A., Lanka, J., Cupriks, L. (2012). Glenohumeral joint muscles strength of the young tennis players. Journal of Human Sport and Exercise, 7, S8-S16.

\section{Corresponding author:}

Andrew L. Shim

Department of Kinesiology \& Human Performance

Briar Cliff University

3303 Rebecca Street

Sioux City, IA. 44154 USA

Phone: 712-279-1780

Email: andrew.shim@briarcliff.edu 